\title{
ORBIFOLD-HODGE NUMBERS OF HILBERT SCHEMES
}

\author{
LOTHAR GÖTTSCHE \\ Dipartimento di Matematica, Università di Pisa \\ Via Buonarroti 2, I-56127 Pisa, Italy \\ E-mail: goettsch@dm.unipi.it
}

1. Introduction. Let $G$ be a finite group acting on a compact differentiable manifold $X$. Then in $\left[D^{*} 1\right],\left[D^{*} 2\right]$ an orbifold Euler number of $X$ is defined by

$$
e(X, G):=\frac{1}{|G|} \sum_{g h=h g} e\left(X^{g} \cap X^{h}\right),
$$

where the sum runs over all commuting pairs in $G$ and $X^{g}$ denotes the set of fixed points of the action of $g$. According to $[\mathrm{H}-\mathrm{H}]$ and $\left[\mathrm{D}^{*} 2\right]$ the formula above can be rewritten as follows: Let $C(g):=\{h \in G \mid h g=g h\}$ be the centralizer of $g \in G$. Then $C(g)$ acts on $X^{g}$ and

$$
e(X, G)=\sum_{[g]} e\left(X^{g} / C(g)\right),
$$

where $g$ runs over a system of representatives for the conjugacy classes of $G . e(X, G)$ is expected to coincide with the Euler number of a crepant resolution of the quotient $X / G$. This fact has been shown in many cases (see e.g. [H-H], [Ro]).

Now let $X$ be a compact Kähler manifold or a Moishezon manifold of complex dimension $d$. The Hodge polynomial $h(X, x, y)$ is defined by $h(X, x, y):=\sum_{p, q} h^{p, q}(X) x^{p} y^{q}$, where the $h^{p, q}(X)$ are the Hodge numbers of $X$. Then, motivated by [V] and [Z], orbifold Hodge numbers $h^{p, q}(X, G)$ are introduced in [B-D] as follows: For any point $x \in X^{g}$, the eigenvalues of $g$ on the tangent space $T_{X, x}$ are roots of unity $e^{2 \pi i \alpha_{1}}, \ldots, e^{2 \pi i \alpha_{d}}$, where $0 \leq \alpha_{j}<1$ and the $\alpha_{j}$ are locally constant functions on $X^{g}$. Let $X_{1}(g), \ldots, X_{r}(g)$ be the connected components of $X^{g}$. For $i=1, \ldots, r$ we put $F_{i}(g)$ equal to the value of $\sum_{j=1}^{d} \alpha_{j}$ on $X_{i}(g)$ and set

$$
h_{g}^{p, q}(X, G):=\sum_{i=1}^{r} \operatorname{dim}\left(H^{p-F_{i}(g), q-F_{i}(g)}\left(X_{i}(g)\right)^{C(g)}\right) .
$$

1991 Mathematics Subject Classification: Primary 14C05; Secondary 14C30.

The paper is in final form and no version of it will be published elsewhere. 
Here $C(g)$ is the centralizer of $g$ in $G$ and $(\cdot)^{C(g)}$ denotes the $C(g)$-invariant part. Then the orbifold Hodge numbers are defined by

$$
h^{p, q}(X, G):=\sum_{[g]} h_{g}^{p, q}(X, G),
$$

where $g$ runs again over a system of representatives for the conjugacy classes of $G$. The orbifold Hodge polynomial of $X$ is $h(X, G, x, y):=\sum_{p, q} h^{p, q}(X, G) x^{p} y^{q}$.

The $X_{i}(g)$ are smooth and thus the quotients $X_{i}(g) / C(g)$ are $V$-manifolds, thus their Hodge numbers are well defined and satisfy $h^{p, q}\left(X_{i}(g) / C(g)\right)=\operatorname{dim}\left(H^{p, q}\left(X_{i}(g)\right)^{C(g)}\right)$. We get therefore the formula

$$
h(X, G, x, y)=\sum_{[g]} \sum_{i=1}^{r} h\left(X_{i}(g) / C(g), x, y\right)(x y)^{F_{i}(g)} .
$$

It is conjectured that, for any crepant resolution $\widetilde{X / G}$ of $X / G$, the orbifold Hodge numbers satisfy $h(X, G, x, y)=h(\widetilde{X / G}, x, y)$. In [B-D] this conjecture is related to the "strong MacKay conjecture" ([B-D] conjecture 5.3, see also [Re]). In [B-D] the conjecture is also shown in several cases and the orbifold Hodge numbers are related to "stringtheoretic" Hodge numbers.

We want to show this conjecture in two cases, where the acting group is the symmetric group, and the resolutions are (related to) Hilbert schemes of points.

(1) Let $S$ be an algebraic surface. Let $G(n)$ be the symmetric group on $n$ letters acting on $S^{n}$ by permuting the factors. Then $S^{n} / G(n)$ is the symmetric power $S^{(n)}$, which has the Hilbert scheme $\operatorname{Hilb}^{n}(S)$ of $n$ points on $S$ as a natural desingularisation via the Hilbert-Chow morphism $\omega_{n}: \operatorname{Hilb}^{n}(S) \longrightarrow S^{(n)}$ (see e.g. [Fo1]). It is easy to see that $S^{n} / G(n)$ is Gorenstein and this resolution is crepant (see e.g. [Be]). In [H-H] it was shown that $e\left(S^{n}, G(n)\right)=e\left(\operatorname{Hilb}^{n}(S)\right)$.

Theorem 1. $h\left(S^{n}, G(n), x, y\right)=h\left(\operatorname{Hilb}^{n}(S), x, y\right)$.

(2) Let $A$ be an abelian surface. Then the symmetric group $G(n)$ acts on $A^{n-1}$ as follows. $A^{n-1}$ is embedded in $A^{n}$ as the subvariety

$$
A_{0}^{n}:=\left\{\left(x_{1}, \ldots, x_{n}\right) \in A^{n} \mid \sum_{i} x_{i}=0 \text { in the group } A\right\} .
$$

Obviously the action of $G(n)$ on $A^{n}$ by permuting the factors restricts to an action on $A_{0}^{n}$. The quotient $A^{n-1} / G$ and its desingularisation can be described as follows: Let again $A^{(n)}$ be the $n$-fold symmetric power of $A$. Then there is a natural morphism $\sigma: A^{(n)} \longrightarrow A$, associating to a 0 -cycle $\sum\left[x_{i}\right]$ its sum $\sum x_{i}$ in the group $A$. Then $A^{n-1} / G(n) \simeq \sigma^{-1}(0)$, and a resolution of $A^{n-1} / G(n)$ is the higher order Kummer variety $K_{n-1}:=\omega_{n}^{-1} \sigma^{-1}(0)$, where again $\omega_{n}: \operatorname{Hilb}^{n}(A) \longrightarrow A^{(n)}$ is the Hilbert-Chow morphism.

Again it can easily be shown that the quotient $A^{n-1} / G(n)$ is Gorenstein and the resolution is crepant $[\mathrm{Be}]$.

Theorem 2. $h\left(A^{n-1}, G(n), x, y\right)=h\left(K_{n-1}, x, y\right)$.

This paper was inspired by the paper [B-D] and a question of one of its authors D. Dais. 
2. The formula for Hilbert schemes. We first introduce some notations and definitions on partitions and the symmetric group.

Definition 2.1. Let $P(n)$ be the set of partitions of $n$. We write an $\alpha \in P(n)$ as $\alpha=$ $\left(1^{\alpha_{1}}, 2^{\alpha_{2}}, \ldots, n^{\alpha_{n}}\right)$, where $\alpha_{i}$ is the number of repetitions of $i$ in the partition $\alpha$, and put $|\alpha|:=\sum_{i} \alpha_{i}$. For $\alpha \in P(n)$ we put $S^{\alpha}:=S^{\alpha_{1}} \times \ldots \times S^{\alpha_{n}}$ and $S^{(\alpha)}:=S^{\left(\alpha_{1}\right)} \times \ldots \times S^{\left(\alpha_{n}\right)}$.

The cycle type $\alpha(g)$ of $g \in G(n)$ is the partition $\left(1^{\alpha_{1}(g)}, \ldots, n^{\alpha_{n}(g)}\right)$, where $\alpha_{i}(g)$ is the number of cycles of length $i$ in the representation of $g$ as a product of disjoint cycles. As usual we denote by $\left(n_{1}, \ldots, n_{r}\right)$ the cycle defined by mapping $n_{i}$ to $n_{i+1}$ for $i<r$ and $n_{r}$ to $n_{1}$.

By [G-S] (see also [Gö1] Thm. 2.3.14) we have

$$
h\left(\operatorname{Hilb}^{n}(S), x, y\right)=\sum_{\alpha \in P(n)}(x y)^{n-|\alpha|} h\left(S^{(\alpha)}, x, y\right) .
$$

In this section we want to show that (2.1) coincides termwise with (1.1). It is elementary that $\alpha(g)$ only depends on the conjugacy class of $g$ and that the map $[g] \mapsto \alpha(g)$ is a bijection from the conjugacy classes of $G(n)$ to $P(n)$.

Lemma 2.2. For $g \in G(n)$ we have $\left(S^{n}\right)^{g} / C(g) \simeq S^{(\alpha(g))}$.

Proof. We see that $\left(S^{n}\right)^{g}$ is the set of all $\left(x_{1}, \ldots, x_{n}\right) \in S^{n}$ such that $x_{i}=x_{j}$ if there exists a cycle of $g$ containing $i$ and $j$. Thus we get $\left(S^{n}\right)^{g}=S^{\alpha(g)}$. The centralizer $C(g)$ acts on the elements of $[g]$ by permuting the cycles of the same length. Therefore it acts on $S^{\alpha(g)}$ by permuting the factors of the $S^{\alpha_{i}(g)}$, and the result follows.

In order to show Theorem 1 we therefore only have to show the following.

Lemma 2.3. For any $g \in G(n)$ we have $F(g)=n-|\alpha(g)|$.

Proof. We can write

$$
S^{n}=S^{\alpha_{1}(g)} \times\left(S^{\alpha_{2}(g)}\right)^{2} \times \ldots \times\left(S^{\alpha_{n}(g)}\right)^{n},
$$

and $g$ operates on $S^{n}$ by permuting cyclically the factors $S^{\alpha_{i}(g)}$ of each $\left(S^{\alpha_{i}(g)}\right)^{i}$. From this it follows easily that $F(g)=\sum_{i=1}^{r} F\left(g_{i}\right)$, where $g=g_{1} \ldots g_{r}$ is the representation of $g$ as a product of disjoint cycles, and the $g_{i}$ are considered as acting on $S^{i}$. Therefore it is enough to show the result in the case that $g$ has cycle type $\left(n^{1}\right)$, and we can assume that $g=(1,2,3, \ldots, n)$.

Let $s \in S$ and $x, y$ local coordinates on $S$ centered at $s$. We denote by $x_{j}, y_{j}$ the pullback of $x, y$ from the $j^{\text {th }}$ factor of $S^{n} . x_{1}, \ldots, x_{n}, y_{1}, \ldots, y_{n}$ obviously give local coordinates of $S^{n}$ near the point $p=(s, \ldots, s) \in\left(S^{n}\right)^{g}$. For $k=1, \ldots, n$ we put

$$
w_{k}:=\sum_{j=1}^{n} x_{j} \cdot e^{2 \pi i k j / n}, z_{k}:=\sum_{j=1}^{n} y_{j} \cdot e^{2 \pi i k j / n} .
$$

Then also $\left(w_{1}, \ldots, w_{n}, z_{1}, \ldots, z_{n}\right)$ form a system of local coordinates, and we get

$$
g\left(w_{k}\right)=e^{-2 \pi i k / n} \cdot w_{k}, g\left(z_{k}\right)=e^{-2 \pi i k / n} \cdot z_{k} .
$$

Therefore the action of $g$ on the tangent space $T_{S^{n}, p}$ is given by

$$
g\left(\frac{\partial}{\partial w_{k}}\right)=e^{2 \pi i k / n} \frac{\partial}{\partial w_{k}}, g\left(\frac{\partial}{\partial z_{k}}\right)=e^{2 \pi i k / n} \frac{\partial}{\partial z_{k}} .
$$

So we get $F(g)=2 \cdot(1+2+\ldots+n-1+0+0) / n=n-1$, and the result follows. 
3. The formula for higher order Kummer varieties. By [G-S] (see also [Gö1] 2.4.17) we have

$$
h\left(K_{n-1}, x, y\right)=\sum_{\alpha \in P(n)}(x y)^{n-|\alpha|} \operatorname{gcd}(\alpha) h\left(A^{(\alpha)}, x, y\right) / h(A, x, y) .
$$

Here we denote for a partition $\alpha=\left(1^{\alpha_{1}}, \ldots, n^{\alpha_{n}}\right)$ of $n$ by $\operatorname{gcd}(\alpha)$ the greatest common divisor of the numbers $k$ with $\alpha_{k} \neq 0$. Again we want to show that (3.1) coincides termwise with (1.1).

Let $g \in G(n)$ of cycle type $\alpha=\left(1^{\alpha_{1}}, \ldots, n^{\alpha_{n}}\right)$. Then $\left(A_{0}^{n}\right)^{g}=\left(A^{n}\right)^{g} \cap A_{0}^{n}=K^{\alpha}$, where we denote by $K^{\alpha}$ the subvariety

$$
K^{\alpha}:=\left\{\left(\left(x_{j}^{1}\right)_{j=1}^{\alpha_{1}}, \ldots,\left(x_{j}^{n}\right)_{j=1}^{\alpha_{n}}\right) \in A^{\alpha} \mid \sum i \cdot x_{j}^{i}=0\right\} .
$$

Then in the proof of Theorem 6 in [G-S] the following is shown:

LEMMA 3.1.

(1) If $\operatorname{gcd}(\alpha)=1$, then $A \times K^{\alpha} \simeq A^{\alpha}$.

(2) In general we have

$$
K^{\alpha}=\coprod_{y \in A(\operatorname{gcd}(\alpha))} K_{y}^{\alpha} .
$$

Here $A(\operatorname{gcd}(\alpha))$ is the set of $\operatorname{gcd}(\alpha)$-division points of $A$ and

$$
K_{y}^{\alpha}:=\left\{\left(\left(x_{j}^{1}\right)_{j=1}^{\alpha_{1}}, \ldots,\left(x_{j}^{n}\right)_{j=1}^{\alpha_{n}}\right) \in A^{\alpha} \mid \sum_{i, j} \frac{i}{\operatorname{gcd}(\alpha)} x_{j}^{i}=y\right\} .
$$

Furthermore we have an isomorphism $K_{y}^{\alpha} \simeq K^{(\beta)}$, where $\beta:=\left(1^{\beta_{1}}, \ldots, n^{\beta_{n}}\right)$ with $\beta_{i}:=\alpha_{\operatorname{gcd}(\alpha) \cdot \beta}$ for all $i$.

It is easy to see that both isomorphisms $A \times K^{\alpha} \simeq A^{\alpha}$ and $K_{y}^{\alpha} \simeq K^{\beta}$ commute with the $G(n)$-action.

As above we see that the centralizer $C(g)$ acts on $A^{\alpha}=A^{\alpha_{1}} \times \ldots \times A^{\alpha_{n}}$ by permuting the factors of each factor $A^{\alpha_{i}}$. So we get

$$
\left(A_{0}^{n}\right)^{g} / C(g)=K^{((\alpha))}:=\left\{\left(\sum_{j=1}^{\alpha_{1}}\left[x_{j}^{1}\right], \ldots, \sum_{j=1}^{\alpha_{n}}\left[x_{j}^{n}\right]\right) \in A^{(\alpha)} \mid \sum_{i, j} i \cdot x_{j}^{i}=0\right\} .
$$

Again by the proof of Theorem 6 in [G-S] we have:

\section{LEMMA 3.2 .}

(1) If $\operatorname{gcd}(\alpha)=1$, then $h\left(A \times K^{((\alpha))}, x, y\right)=h\left(A^{(\alpha)}, x, y\right)$.

(2) In general we have

$$
K^{((\alpha))}=\coprod_{y \in A(\operatorname{gcd}(\alpha))} K_{y}^{((\alpha))} .
$$

Here

$$
K_{y}^{(\alpha)}:=\left\{\left(\sum_{j=1}^{\alpha_{1}}\left[x_{j}^{1}\right], \ldots, \sum_{j=1}^{\alpha_{n}}\left[x_{j}^{n}\right]\right) \in A^{(\alpha)} \mid \sum_{i, j} \frac{i}{\operatorname{gcd}(\alpha)} x_{j}^{i}=y\right\} .
$$

Furthermore we have an isomorphism $K_{y}^{((\alpha))} \simeq K^{((\beta))}$, where $\beta:=\left(1^{\beta_{1}}, \ldots, n^{\beta_{n}}\right)$ with $\beta_{i}:=\alpha_{\operatorname{gcd}(\alpha) \cdot \beta}$ for all $i$.

If we put these results together, it is enough (for the proof of Theorem 2) to show: 
Lemma 3.3. For the action of $g$ on $A_{0}^{n}$ we have $F(g)=n-|\alpha|$.

Proof. The action of $G(n)$ on $A_{0}^{n}$ is just the restriction of the action on $A^{n}$. Therefore in the same way as in the proof of Lemma 2.3 we can assume that $g=(1,2, \ldots, n)$. Let again $\left(x_{i}, y_{i}\right)$ be pullbacks of local coordinates from the $i^{\text {th }}$ of $A^{n}$ near a point

$$
p \in\left(A_{0}^{n}\right)^{g}:=\{(a, \ldots, a) \mid a \in A(n)\} .
$$

We can assume that $a$ is the neutral element of the group $A$. Now we define local coordinates $w_{1}, \ldots, w_{n}, z_{1}, \ldots, z_{n}$ on $A^{n}$ by the same formulas as in the proof of Lemma 2.3. Then the subvariety $A_{0}^{n} \subset A^{n}$ is near $p$ defined by the equations $w_{n}=0, z_{n}=0$, and $w_{1}, \ldots, w_{n-1}, z_{1}, \ldots, z_{n-1}$ are local coordinates on $A_{0}^{n}$. We get as above $g\left(\frac{\partial}{\partial w_{j}}\right)=$ $e^{2 \pi i j / n} \frac{\partial}{\partial w_{j}}, g\left(\frac{\partial}{\partial z_{j}}\right)=e^{2 \pi i j / n} \frac{\partial}{\partial z_{j}}$. Therefore we obtain $F(g)=2 \cdot(1+2+\ldots+(n-1)) / n=$ $n-1$, and the result follows.

\section{References}

[B-D] V. V. Batyrev and D. Dais, Strong McKay correspondence, string-theoretic Hodge numbers and mirror symmetry, preprint 1994.

[Be] A. Beauville, Variétés kähleriennes dont la première classe de Chern est nulle, J. Differential Geom. 18 (1983), 755-782.

[D*1] L. Dixon, J. Harvey, C. Vafa and E. Witten, Strings on orbifolds I, Nuclear Phys. B 261 (1985), 678-686.

[D*2] L. Dixon, J. Harvey, C. Vafa and E. Witten, Strings on orbifolds II, Nuclear Phys. B 274 (1985), 285-314.

[Fo1] J. Fogarty, Algebraic families on an algebraic surface, Amer. J. Math. 10 (1968), 511521.

[Fo2] J. Fogarty, Algebraic families on an algebraic surface II, Picard scheme of the punctual Hilbert scheme, Amer. J. Math. 96 (1974), 660-687.

[Gö1] L. Göttsche, The Betti numbers of the Hilbert schemes of points on a smooth projective surface, Math. Ann. 286 (1990), 193-207.

[Gö2] L. Göttsche, Hilbert schemes of zero-dimensional subschemes of smooth varieties, Lecture Notes in Math. 1572, Springer Verlag, Berlin, Heidelberg, New York, 1994.

[G-S] L. Göttsche and W. Soergel, Perverse sheaves and the cohomology of Hilbert schemes of smooth algebraic surfaces, Math. Ann. 296 (1993), 235-245.

[H-H] F. Hirzebruch and T. Höfer, On the Euler number of an orbifold, Math. Ann. 286 (1990), 255-260.

[Re] M. Reid, The MacKay correspondence and the physicists' Euler number, Lecture notes given at Univ. of Utah and at MSRI 1992.

[Ro] S.-S. Roan, Orbifold Euler characteristic, Inst. of Math. Acad. Sinica, preprint 1993.

[V] C. Vafa, Sting vacua and orbifoldized LG Models, Modern Phys. Lett. A 4 (1989), 1169-1185.

[Z] E. Zaslow Topological orbifold models and quantum cohomology rings, Comm. Math. Phys. 105 (1993), 306-331. 